\title{
Bemerkungen zu der Abhandlung Belogolowys über Parasitismus von Embryonen und die dabei entstehenden bösartigen Geschwülste.
}

\author{
Von
}

Wilhelm Roux.

Eingegangen am 15. Dezember 1917.

Die vorstehende bedentende Abhandlung mit dem Titel: $D i e$ Einwirkung parasitären Lebens auf das sich entwickelnde Amphibienei (den Laichball)* ging zwei Monate vor dem Beginne des Krieges bei der Redaktion des Archivs ein. Durch den Kriegsbeginn wurde der bereits begonnene Satz unterbrochen, und die Arbeit ruhte tiber drei Jahre in der Druckerei zu Leipzig.

Die lange Dauer des Krieges und der bevorstehende Verlagswechsel des Archirs machte nun doch noch die Veröffentlichung ohne eine Korrektur des russischen Autors nötig. Die eingesandten Abbildungen sind vollkommen getreu reproduziert.

Die Abhandlung schildert and dentet die Ergebnisse eines neuen Experimentes: der Einpflanzung nackter Kröten- und Frosch-Moralae, -Blastulae, -Gastrulae und junger Neurulae in die Banchhöhle von erwachsenen Fröschen und Kröten.

BELOGOLOWY will die Wirkung des parasitischen Lebens auf das sich entwickelnde Ei, aber auch auf den Wirt ermitteln; er sieht den Parasitismus als eine Verbesserung der Lebensumstände an und meint, daß eine solche die Zahl der Gestaltungsmerkmale des Parasiten vermindern, also den Bau desselben vereinfachen müsse, daß eine' direkte Anpassung des einzelnen Parasiten, bzw. seiner Teile an das günstigere Medium auch in diesen neuen Verhältnissen stattfinden werde. Von dieser Auffassung aus deutet er die Beobachtungsergebnisse auf Grund reicher vergleichend-biologischer Kenntnisse.

Seine Befunde sind im Speziellen von großer Mannigfaltigkeit. Deshalb sowie weil die fixierten und mikrotomierten Beobachtungsstadien sich auf den großen Zeitraum von 4-6 Monaten verteilen, ist die Deutung der Befunde trotz der sehr großen Zahl der Versuche recht schwierig. 
Diese Schwierigkeit betrifft zunächst schon die Ableitung der zwar an sich sichtbaren, aber nicht gesehenen Bildungsvorgänge aus den fixierten Stadien; noch mehr betrifft sie natirlich die allgemein biologische und die kansale Dentung des Gesehenen sowie des ans ihm Abgeleiteten. Es wird daher nicht zu verwandern sein, wenn Leser dieser Arbeit und später die Nachuntersucher Manches anders deaten werden, als BELOGOLOWY selber.

Aber schon die unangreifbaren tatsächlichen Befunde BELOGoLOWYs sind von großem Werte sowohl für die Entwicklungsmechanik wie für die allgemeine Biologie und für die Geschwalstlehre.

Viele Ergebnisse haben auch nahe Beziehungen zu bisherigen Beobachtungen und Auffassungen der Entwicklungsmechanik. Der AnschluB an diese soll hier gleich bergestellt werden, da der Antor dies unterlassen hat, wohl weil seine Interessen und daher auch seine Dentungen auf dem Gebiete des Parasitismus und der allgemeinen Biologie liegen.

Die direkten Befunde können in vier Gruppen geteilt werden. Sie betreffen:

1) die Erkenntnis, daß die typische Entwicklung des Anureneies durch die Implantation in die Banchböhle eines erwacbsenen Amphibiums, also durch die neue Umgebung, hochgradig verändert, oft ganz aufgegeben wird, ersteres um so mehr, in je frtherem Entwicklungsstadium die Implantation erfolgte;

2) daß unter diesen Umständen eine Auflösung des Embryo in getrennte, fitrsich weiterlebende Zellkomplexe und einzelne Zellen erfolgt;

3) daß diese isolierten lebenden Teile sich in sehr verschiedener Weise verhalten können:

a) indem sie Produkte liefern, die noch normalen Gewebe- und Organbildungen entsprechen oder ihnen ähnlich sind: atypisch gestaltete Knorpel-, Knochen-, Drüsen-, Muskelstiicke, Eier, und annäbernd normal gestaltete Chorda dorsalis und Gehirnblasen;

b) indem sie rom Typischen ganz abweichende Produkte liefern: Cysten, Plasmodien und einzelne Zellen verschiedener Art, besonders sarkomartige Zellen;

4) daß die isolierten Embryoteile in eigenartige Beziehung zum Wirt treten, indem

a) die sarkomartigen Zellen in die Gewebe des Wirts eindringen, dessen Organe zerstören und den Wirt regelmäßig nach 5-6 Monaten töten: also sicherer Nachweis der Entștehung bösartiger Geschwliste aus implantierten embryonalen Zellen; 
b) indem die embryonalen Zellen das Wachstam und sogar die Regeneration von Organen des Wirts (der Leber) anregen.

Die Auflösung des Embryo in der Leibeshöhle leitet BeLoGoLowy davon ab (S. 667), daß die Zellen des implantierten Organismus außerhalb desselben "passende Lebensverbältnisse * finden. Eine noch weiterhin sich erhaltende Abgrenzung des Individuums werde infolgedessen unmöglich, was sich gleich dadurch kund tut, daß das Ektoderm, welches dem Unterschied der inneren und äußeren Lebensverhältnisse entsprach, verschwindet: es zerfällt in Zellen, welche in den umgebenden Raum auswandern.

Diese Begründung teile ich nicht. Ich glaübe nicht, daß die Erhaltung des normalen Ektoderms allein durch den Unterschied des inneren und äußeren Mediums des Embryo bedingt wird; dann müßte sich schon normalerweise das Fruchtwasser des Amphibieneies, noch mehr das Fruchtwasser des Sängetiereies im Laufe der Embryogenese in typischer Weise verändern, um immer zu der typisch fortschreitenden Differenzierung der Epidermis passen zu können.

Ich glaube auch nicht, daß die Auflösung des epithelialen Verbandes des Ektoderms dadurch bedingt wird, daß in der Bauchböhle fur das Zellenleben des Ektoderms zu gut passende Bedingungen vorhanden sind.

BeLogolowy leitet dagegen aus seiner Anffassung eine neue Definition des $*$ Ganzen * des Lebewesens ab, indem er (S. 667) sagt: - Wir muissen der gewohnten Vorstellung entsagen und den Organismus als einen von allen Seiten begrenzten Teil des Raumes ansehen, welcher Medien enthält, die für sein Leben unermeßlich günstiger sind als die ihn umringende Außenwelt.

Dagegen ist zu bedenken, daß das sich entwickelnde Lebewesen dieses innere Medium selber erst produziert hat. Daher sind die spezifisch strukturierten Teile wesentlicher als das von ihnen prodazierte innere Medium.

In dem weiteren Verhalten der ïberlebenden isolierten Teile sieht BELOGOLOWY (S. 665) in teleologischer Auffassung *Anpassungsversuchex:

1) in den Cysten und soliden kugligen Gebilden: » verfehlte Versuche*, neue mehrzellige Organismen auf Grund einer unmittelbaren Modifikation der Entwicklung des parasitären Laichballs selbst zu bilden;

2) in den Plasmodien: xerfolgreicherere Versuche^, neue biologische Zyklen von plasmodialem Charakter zu bilden; 
3) in den einzelligen Gebilden: »am besten gelungene Versuche*, Zyklen in Form verschiedener einzelliger Individuen zu bilden.

Die Fähigkeit zu diesen Vorgängen leitet er ans der Erweckung schlummernder Ahnenplasmen, atavistischer Gene ab.

Es sei zunächst anf einige Tatsachen der Entwicklnngsmechanik hingewiesen, nach welchen die in Gruppe 1 erwähnten Beobachtungsergebnisse nicht mehr so eigenartig erscheinen. In den Jahren 1882 bis 1884 hatte ich Froscheier angestochen und dadurch zunächst unbestimmt begrenzte Teile, später präzis eine der zwei oder vier ersten Furchungszellen abgetötet. Danach batten sich die tiberlebenden Eistuicke weiter entwickelt; letzteren Falles bildeten die isolierten uberlebenden Furchungszellen halbe oder Dreiviertelembryonen. Diese Árt der Bildung der einzelnen Stikcke des Embryo durch Selbstdifferenzierang der Teile nannte ich Mosaikarbeit. Zwei Jahre nach meiner ersten Publikation ${ }^{1)}$ veröffentlichte CHabRY (1887) entsprechende Anstichversuche an Ascidieneiern mit ähnlichem Ergebnis. Auch vou mir durch Einschnitt an Gastrulae und Blastulae gebildete zungenförmige Lappen entwickelten sich in typischer Weise weiter. Seitdem sind viele weitere Tatsachen der Selbstdifferenziẹrung embryonaler Zellen und Organanlagen ermittelt worden von Spemany, Braus, Fischel u. a., so daß die Befunde Belogolowys der Bildung von Chorda, Knorpel, Knochen, Dritsen, Gebirnblasen nichts Besonderes darstellen.

Im Jahre 1894 (Lit. 1894b) löste ich die Frosebmorula durch Zerreißen in halbprozentiger Kochsalzlösung mechanisch in ibre Zellen auf und beobachtete danach, daß viele Furchungszellen direkte Näherungswirkungen anfeinander austiben, indem sie "schrittweise $\propto$ in gerader Richtung gegeneinander wanderten, ein Geschehen, das ich Cyto-

1) Beitrag I zur Entwicklungsmechanik des Embryo. Zeitschr. f. Biologie München 1885 (auch in Gesamm. Abh. Bd. II). Diese erste Publikation berichtet auf 25 Seiten bereits über bestimmt lokalisierte Eianstiche bzw. -Anschnitte in allen Stadien von der Befruchtnng bis zum Embryo (Neurula). Ein Abzug davon warde Herrn Prof. Poucher im Juni 1885 iibersandt, der sich sobriftlich dafür bedankte. $Z_{w e i}$ volle Jahre danach publizierte dessen Schitler CHABRX gleichfalls eine Arbeit liber Anstichversuche, und zwar an Ascidieneiern, jedoch mit der Bemerkung, daß er damit einen nenen Weg der Forschung betreten habe. Meine zweite Publikation ersehien 1887 im Anatom. Anz. Nr. 25, dio dritte 1888 (s. Lit.). Ich habe diese Sachlage 1894 im Anatom. Anz., Bd. 9, S. 260 dargelegt, und BARFURTH hat sie in seinen vielen, sehr verbreiteten Berichten und sonstigen Schriften wiederbolt erwähnt. Gleichwohl haben anßer ansländischen Autoren auch ein paar (gegnerische) deutsche Autoren (O. Hertwig, M. Nussbaum) die Priorität Chabry zugeschrieben. 
tropismus nannte. An die so entstandene Beruhrung schloß sich oft flächenhafte Vereinigung der Zellen (Cy tarme), dann folgte mannigfache Umordnung durch Zellengleiten (Cytolisthesis), beides bis zur Bildung gerundeter Zellkomplexe, letztere oft sogar mit glatt, also ungeknickt uber die Zellgrẹnzen weggehender Oberfläche des Komplexes (Bildung ageschlossener"Komplexe, die den Plateauschen Gesetzen der Blasenspannung widerspricht). Diese Geschlossenheit beruht nach meiner Auffassung einfach darauf, daß die Oberflächenspannung an den Berührungsflächen der Zellen in diesen Fällen Null geworden, also ganz geschwunden ist, so daß allein noch die Spannung der äußeren Oberfläche des Komplexes denselben nun als einheitliches weiches Gebilde rundet. Dasselbe sieht man z. B. auch noch an Klimpchen abgekratzten Flimmerepithels des Froschrachens beim Verweilen in halbprozentiger Kochsalzlösung.

Der Komplexbildung folgt oft Wiederaustritt bzw. Ausstoßung mancher Zellen (Cytochorismus), und beim Absterben geschah manchmal vollkommene Wiedertrennung aller Zellen unter Wiederrundung derselben (Framboisia minor). Nach Einwirkung von Borsäurelösung auf die unverletzten Eier sah ich gleichfalls Framboisie und danach Ablösung der Epithelzellen, zuerst nur im Bereiche der Medullarplatte (Amyelie), entstehen; später geschah dasselbe auch im Entoderm und im Mesoderm. (Lit. 1895, II. S. 152, 887.)

Fast alles dies ist in BeLOGoLowys Versuchen auch erkennbar, wenn auch nicht so exakt und analytisch, nicht an je einer isolierten Zellgruppe in flüssigem Medium für sich allein und nicht durch direkte Beobachtung unter dem Mikroskop feststellbar wie in diesen vanalytischen* Versuchen. In ihnen geschah aber alles dieses ohne Parasitismus und jedenfalls in nicht besonders nahrhaftem Medium. Der Parasitismus als solcher ist also dazu nicht nötig, und die Erscheinungen stellen daher anch keine Anpassungen an ihn dar.

C. Herbst machte die wichtige Entdeckung, daß in kalklosem Seewasser die Furchungszellen der Seeigel sich trennen, und Driesch isolierte die Furchungszellen des Seeigeleies durch Schütteln. Die isolierten ersten Zellen entwickelten sich zu normalen Ganzbildungen.

BeLogolowys Auffassung von der auflösenden Wirkung eines •passenden،, also doch wohl gut nährenden Mediums auf das äußere Epithel stimmt auch nicht recht tuberein mit den Ergebnissen der Explantationsversuche Carrels, Burrows, Braus' a. a. Denn das Epithel embryonaler wie erwachsener Organe : framboisierte nicht 
in Medien, in welchen diese Teile Wochen, sogar Monate lang weiter lebten bzw. sich weiter entwickelten.

Aus allen diesen Erfahrungen schließe ich, daß die den epithelialen Verband durch Framboisie auflösende Wirkung erstens nur an solchen Epithelien möglich ist, deren Zellen noch nicht so fest zu eckiger Gestalt differenziert sind, daß diese anch nach der mechanischen Isolierung noch erhalten bleibt, sondern daß die Zellen sich nach dieser Isolierung noch abrunden oder ganz runden, wie dies die Ektodermzellen sehr junger Embryonen und noch von Erwachsenen, z. B. die Leberzellen (auch des Menschen), die Zellen des Rachenepithels des Frosches tun. Und zweitens, daß beim Vorhandensein dieser Zelleigenschaft zar Auflösung des epithelialen Verbandes schon eine Einwirkung zureichend ist, welche die Oberflächenspannung an den Seitenflächen der Epithelien in frühester Stärke wieder herstellt. Das kann durch sehr verschiedenartige Wirkungen, auch durch schädigende, nicht bloß durch gut ernährende Medien geschehen (1896 b, S. 70). Auch Kontraktion der Zellen kann dasselbe bewirken.

In BELOGOLOWYs Versuchen löste sich das gefurchte Froschei auf wie die Morula in Herbsts Medium, welches keineswegs als . Verbesserung * eines Lebensumstandes aufgefaßt werden kann.

Die kugligen Hoblbildangen, die Cysten, sind nach meiner Auffassung Produkte der Erhaltung einer sehr allgemein verbreiteten niederen, nicht besonders spezifizierten Gestaltungspotenz des Epithels, die auch in anderen abnormen Fällen von isolierten Epithelstilucken vorkommt und wohl nur eine Folge der polaren Differenzierung und der daher einseitigen Absonderung der Epithelzellen sowie ihrer spezifischen Eigenschaft, sich mit den "Seitenflächen * zu vereinigen (der typischen Cytarme), ist. In der Bildung der soliden kugligen Zellhau fen erkenne ich nur Wirkungen der *Oberflächenspannung*, welche so lange wirkt, als die Zellen noch flussige gespannte Oberflächen haben. Die Zellen vereinigen sich hier zn rundlichen Komplexen wie die isolierten Furchungszellen, wie die Zellen der abgekratzten Stuckchen vom Rachenepithel des erwachsenen Frosches in halbprozentiger Kochsalzlösung. Von besonderem Interesse ist die Bildung von Kapseln aus Epithel, Knorpel, Bindegewebe um diese and andere abnorme Bildungen und die oft große Dicke dieser Kapsèln, noch mehr die Bildung von drei dicken Kapseln um einen Zellkomplex. Der Meinung des Autors, daß darin Anpassung zur Bildung neuer Individuen vorliegt (S. 595, 610,614) kann ich nicht zustimmen. Fur irgendeine gedachte, das Leben des umschlossenen Gebildes 
erhaltende Funktion sind diese Kapseln oft viel zn dick. Ich leite sie einfach von (vielleicht chemischer) Erregung gestaltender Reaktiónsmechanismen ab, und finde es sehr lehrreich, daß von abnormen Gebilden gleich so regelmäßig gebildete, auch konzentrisch geschichtete Kapseln produziert werden, also ohne spezielle vererbte Determinationen gebildet werden können ${ }^{1}$ ).

Die noch weiter differenzierten Bildungen, die Stiicke von Knorpel, Knochen, Chorda, Myotome, Drïsen, Gehirnblasen, sind nur Beispiele der lange erwiesenen Selbstdifferenzierungsfähigkeit embryonaler Zellen der Amphibien; diese Fähigkeit hat die Lehre von den Mißbildnngen auch an Säugetieren erwiesen, so z. B. die Bildung des Hemitherium anterius (Lit. 1895, II. S. 446, 828; 1902, S. 254) des Kalbes (Roux). Die Bildung von Schwanzmyotomen beim Fehlen des Schwanzes bekundet ebenso wie die Bildung von Kopfmyotomen beim Fehlen des Kopfskeletts die hochgradige Selbständigkeit dieser Bildungen.

Daß diese T'eilgebilde sich nicht zu typisch gestalteten ganzen Organen differenzierten, ist verständlich, da ihre Bildungszellen aus dem natïrlichen Zusammenhang und damit auch aus der typischen Lage gebracht waren, so daß die Mitwirkung der normalen nachbarlichen Bildungszellen fehlte. Ich habe ausgesprochen, daB je mehr Bildungszellen von Mosaikeiern in ibrer normalen Lage beieinander sind, um so weiter die typische Selbstdifferenzierung des Zellenkomplexes geht, zumal wenn seine Zellen von derselben Furchungszelle abstammen. Denn Selbstdifferenzierung ist nur ein stopographischkausaler Begriff ; die Differenzierung jedes "Selbstdifferenzierungsbezirkes $\propto$ geschieht durch differenzierende Wirkung seiner Unterteile aufeinander, wie jede Differenzierung durch Wirkung von Teilen aufeinander bewirkt wird. Deshalb entsteht aus dem Komplex aller Nachkommen einer Halbei-Blastomere des Froscheies ein wohlgebildeter halber Embryo, der sich bei gentigender Ernährung bis zum Ablauf der präfunktionellen Periode I erhalten kann wie ein Akormus als Zwillingsgebilde (Lit. 1911). Formale Abweichungen finden sich

1) Es ist auch an die Mißbildungen und Degenerationserseheinungen zu erinnern, welche J. Grochmalicki und besonders Laura Kavfmann bei der massenhaften Entwicklung von Salamandern im Uterus beobachteten. Letztere sah a. a. anch Verlagerung des Keimepithels unter die Haut und in die Darmwand mit nachfolgender sehr starker Bildung von Eizellen in diesen fremden Geweben (dies Archiv. Bd. 28 und 37), was Belogolow rs beztiglichem Befunde entspricht. 
an dem Hemiembryo nur an der offenen Seitenfläche, wo der Gegendrack der Nachbarschaft fehlt.

In diesen Teilbildungen BeLogoLowys sehe ich teils ein Stehenbleiben auf niederer Stufe infolge von Hemmung der weiteren höheren Entwicklungsfähigkeit durch die Isolation, weil zur weiteren Differenzierung nötige differenzierende Einwirkungen anderer Teile fehlen; und in mancher abnormen Beschaffenheit sind außerdem noch direkte Störungen durch abnorme Einwirkungen zu erkennen. Das Anpassungsprinzip ist zur Entstehung dieser Gebilde nicht nötig; sie stellen somit keine *neuen Individualitäten *, sondern im Gegenteil nur »lebenden Individuen-Detritus * (Roux) dar.

Die Selbstdifferenzierung oder autogene Differenzierung erscheint BeLogoLowY aber seine Betrachtungén störend, denn er sagt S. 662: - Leider stoßen wir auch hier anf einige Daten, welche eine gewisse Determination der Organentwicklung vermuten lassen, denn anders wäre das Entstehen von Chorda, Knorpeln, Knochen, Geschlechtszellen im Granulationsgewebe außerhalb des [NB. früheren unzerstiickelten] Embryo schwer zu erklären. Nur das Vorhandensein determinierter Zellen erklärt diesen Wirrwar der Bildungen.* Für meine Auffassung ist der Wirrwar, wenn man von einem solchen reden will, nicht durch die Reste typischer Bildangen, sondern durch die vielfachen, abnormen Bildungen verursacht.

Diese letzteren Bildungen beurteilt BeLogolowy (S. 663) als Wirkung des umgekehrten biogenetischen Grundgesetzes. Dementgegen vertrete ich schon seit einigen Dezennien die Auffassung, daß Naturgesetze nur menschliche Formulierungen für ermittelte beständige Wirkungsweisen sind und daher nicht wirken (1912, S. 271). Außerdem ist das sog. biogenetisehe Grundgesetz nach meiner Meinung kein * Gesetz des Wirkens *, sondern nur eine $\gg$ Regel des 'Vorkommens * (s. 1912, Terminologie S. 64). Eine wirkliche Umkehrung der durch diese Regel bezeichneten Reihenfolge von Bildungsvorgängen ist zwar von einigen Forschern vertreten, aber noch nicht prinzipiell erwiesen worden.

BeLogolowy vermutet, daß alle diese Teilgebilde eine Funktion haben. Von der anf Grund vieler Mißbildungen und normaler Gestaltungsvorgänge von mir anfgestellten afunktionellen Gestaltungsperiode I scheint er keine Kenntnis gewonnen zu haben. Die großen Knorpelmassen z. B. können in einem muskellosen embryonalen Gebilde unmöglich eine ihrer Größe entsprechende Stlitzfunktion ausuben, also nicht durch solche herangebildet sein. 
In den einzelligen Gebilden gieht Belogolowy neue'Individualitäten, welche direkte Anpassungen höchsten Grades darstellen, da das parasitische Leben bekanntlich mit Vereinfachung der Organisation verbunden ist. Ich bezweifle aber, daß diese vereinfachende Wirkung in qualitativ neuen Fällen direkt stattfindet und beurteile die in den Versuchen aufgetretene Vereinfachung anders.

Die Furchungszelle ist in ihren aktivierten Potenzen zunächst fast nur eine Amöbe, die aber potentielle Fähigkeiten zur weiteren höheren Entwickelung besitzt. Diese letzteren Fähigkeiten können für sich allein gestört werden, ohne daß die Zelle ihr Leben und damit ihre Amöbeneigenschaft einbüßt. Manche Zellen der von mir zerrissenen Morula benahmen sich als frei bewegliche, nach allen Seiten laufende Amöben, mit protoplasmatischen und paraplasmatischen Pseudopodien.

Statt Weckung zur Anpassung geeigneter atavistischer Potenzen (konkreter gesagt: atavistischer Gene, Determinationsfaktoren, s. 1912, S. 96) haben wir hier als erstes ursächliches Geschehen in den isolierten Zellen abnorm lange dauernde Alleinerhaltung der in normaler Weise zuerst erregten, dem niedersten. Protistenstadium entsprechenden Potenzen, dazu kommt die Hemmung oder Vernichtung der weiteren typischen Gestaltungspotenzen.

An diese Erhaltung niedersten einfachen Zustandes schließen sich aber nach BELogoLow Ys eingehender Schilderung Umwandlungen der Zellen an, die anscheinend direkte Anpassungen an das parasitische Leben im Sinne BeLogolowys darstellen, da die betreffenden Gebilde: die Plasmodien und sarkomähnlichen Zellen die ganze Zeit, 5 Monate lang, mitlebten. Nicht aber ist die Isolation an sich schon als durch solche Anpassung bewirkt aufzufassen, obschon mit dem parasitischen Leben verbundene Umstände die Ursache der Isolation waren, wie ich das oben (S. 684) erörtert habe.

Statt direkter Anpassungen c, die nach BELOGOLOWY sihren Zweck erreichen. (S. 632), sehe ich in dem slebenden Detritus* zunächst nur durch die abnormen Einwirkungen veranlaßte Variationen sei es mit nenen Eigenschaften oder infolge von Weckung atavistischer Eigenschaften. Wie immer bei neuen Variationen bleiben (Lit. $1881 \mathfrak{u}$. 1912, Terminologie S. 215, 424: zilchtende Teilauslese) nur diejenigen lebenden Teile erhalten, die erstens in sich selbsterhaltungsfähig, also assimilationsfähig usw. $(1915, \mathrm{~S} .175)$ und außerdem in den umgebenden Verhältnissen, also auch in der Konkurrenz mit den anderen 
Teilen um Nahrung und Raum, >danerfähig * sind 1). Die nicht dauerfähigen Gebilde sterben und werden zumeist anfgefressen, schwinden somit. Je länger das ganze Geschehen dauert, um so mehr werden die dauerfähigen Gebilde sich aufhäufen, und bei gentigend langer Dauer werden nur noch »lebenddauerfähige * Gebilde vorhanden sein, abgesehen von den wenigen todwiderstandsfähigen* Resten. Die noch lebenden neuen Gebilde scheinen dann »Anpassungen * zu sein, obschon sie bloß Überbleibsel zufälliger (zweckloser) Produkte zu sein brauchen.

Es wird noch vieler Versuche bedürfen, um die kansale, bzw. die biologische Bedeutung der reichen Beobachtungsergebnisse BeLoGolowys zu ermitteln und festzustellen, ob (ev. welche) wirkliche direkte Anpassungen bei ihrer Bildung mitgewirkt haben.

Weitere Versuche mit Implantation noch mit der Gallerthulle oder mit der innersten Haut derselben bekleideter Eier werden weiteres lehren und uns wohl auch uber die wichtigen chemisch-physikalischen Funktionen dieser Htillen aufklären, die wir bis jetzt nur als mechanische Schutzhullen und die Gallerte als Sauerstofferreger (Roux) kennen (1895, II. S. 165, 100).

Von großer Wichtigkeit ist die von BeLOgoLowy entdeckte Tatsache (S. 616, 636, 646, 651), daß viele Zellen der Amphibienmorụla (und -Blastula?), wenn so junge Eibälle in die Bauchhöhle erwachsener Amphibien implantiert worden sind, sich $\mathrm{zu}$ bösartigen Geschwtulsten entwickeln, welche den Wirt in 5 Monaten töten, währepd die von den Pathologen oft implantierten Sticke und Zellen älterer Embryonen im Gegenteil. stets von den Zellen des' Wirtes cytophagiert wurden. Das sichtbare Vorgehen der sark omartig gewordenen bösartigen Zellen entspricht im wesentlichen dem vom Menschen her bekannten Verhalten der Sarkomzellen, und ihre Bildung ist so massenhaft, daß sie bis ein Funftel vom Raume des Wirtes einnehmen. Diese zerstörende Tumorbildung fand auch bei Implantation von Frosch-Laichbällen in Fische (Karausche) statt (S. 658). Es wird durch analytische Versuche zu ermitteln sein, ob die so jung isolierten Furchangszellen an sich schon diese zerstörenden Eigenschaften auf die älteren Gewebe haben, oder ob diese Eigenschaften erst durch chemische Alteration oder durch besondere Erregung entstehen bzw. aktiviert.werden.

1) Ich sage (1881) dauerfähig, dauerfordernd statt zweckmäßig nnd dauermäßig (RIBBERT), da ersteres rein objektiv die sachliche Eigenschaft bezeichnet, während letztere beiden teleologisch sind; dauermäßig heißt der Dauer sangemessen`, setzt also eine zweckmäBige Handlung voraus. 
Da ich (1888) in verschiedenen Keimblättern und Organen schon weit entwickelter Froschembryonen nicht differenzierte Zellen mit allen sichtbaren Merkmalen der Furchungszellen aufgefunden habe, so ist nach den Befunden BeLogolowrs nunmehr die Wahrscheinlichkeit näher gerückt, daß auch diese Zellen, wenn sie später noch erhalten sind und zufällig durch öftere Insulte einer solchen Stelle erregt, die. umgebenden Gewebe aber geschwächt werden, Geschwülste bilden können, was dann eine Bestätigung meiner-hypothetischen Kombination von Consheims Theorie mit der Insulttheorie geben witrde (1895, I. S. 302 ; II. S. 495 n. f.).

Als ich Schnitte dieser Embryonen 1894 in Wien auf der Naturforscherversammlung den Pathologen und Anatomen demonstrierte (1894 a, S. 358), sprach KoELliker seine Verwunderung dartiber aus, daB ihm solche Zellen in seinen vielen Tausend Präparaten nie vorgekommen waren. Das rührte wohl davon her, daß er nur in der freien Natur befruchtete normale Eier benutzte. Ich habe solche Zellen nur nach abnorm verzögerter Laichung, also in Embryonen, welche aus überreifen Eiern entstanden waren, sowie nach Anstich von Eiern gefunden; Ersteres veranlaßte mich die Vermutung $z u$ änßern, daß vielleicht Menschen, welche ans sehr verspätet befruchteten Eiern entsianden sind, dadurch zur Bildung bösartiger Tumoren disponiert sind (1895, II. S. 496). Es empfiehlt sich daher, darauf bezügliche Statistiken zu machen; and die jetzigen Kriegsverhältnisse bieten dazu eine wohl nie so präzis und reich wiederkehrende Gelegenheit $\left.{ }^{1}\right)$. Andererseits empfiehlt es sich, die Implantationsversuche BELoGolowYs mit Eiern des Moralaund Blastulastadiums von und an Säugetieren zu wiederholen; anch Kreuzungen von Amphibien und Säugern oder von Vögeln und Säugern geben dabei vielleicht interessante Resultate und Erkenntnis.

Von Interesse ist auch die neue Bestätigung der Erfahrung von Born, Harrison, Braus a. a., daß bei embryonalen Amphibien die biochemische Differenz für die Erhaltung des Implantates nicht so nachteilig ist wie bei erwachsenen Sängern und dem Menschen. Dies ergab sich daraus, daß gekreuzte Implantationen zwischen Kröten und Fröschen dieselben Ergebnisse lieferten wie die Implantationen innerhalb der Spezies. Vielleicht ergeben daher auch sembryonale* Kreazungsimplantationen zwischen Amphibien und Sängern ein belehrendes Resultat.

1) Bezüglich der Wirkung der Überreife der Eier sei noch darauf hingewiesen, daß jüngst Leo ADLER ans iberreifen Froscheiern Embryonen mit typischer Basedow-Str a ma sich entwickeln sah (dies Archiv. Bd. 43. S. 354; 
Schließlich sei noch auf die wichtige, wenn auch noch nicht gesicherte Angabe BELogolowys hingewiesen, daß Zellen der implantierten jungen Embryonen das Wachstum und die Regeneration derjenigen Organe des Wirts anregen, an denen sie angewachsen sind (S. 615, 564). Der Autor scheint meiner Ableitung des Regenerationsvermögens von einem in den Organzellen enthaltenen somatischen « Keimplasma (1881, S. 178; 1895, I. S. 344, II. S. 905; 1913, S. 14) nahe zu stehen.

Roux, Wilhelm.

\section{Literatur.}

1881. Der Kampf der Teile im Organismus. Leipzig, anch in 1895, Bd. I.

1885. Beiträge zur Entwicklungsmechanik des Embryo. Nr. 1. Zeitschr. für Biologie. München. Bd. 21 , auch in 1895 , Bd. II.

1887. Über Selbstdifferenzierung der Furehungszellen. Anatom. Anzeiger. Nr. 25.

1888. Beiträge zur Entwicklungsmechanik des Embryo. Nr. 5. Über die kiunstliche Hervorbringung halber Embryonen durch Zerstörung einer der beiden ersten Furchungszellen, 'sowie über die Postgeneration der fehlenden Körperhälfte. Vırchows Archiv. Bd. 114.

1894. a) Tageblatt der Versamml. Deutscher Naturf. u. Ärzte. S. 358.

b) Über den Cytotropismus der Furchungszellen des Grasfrosches. Arch. f. Entwicklungsmechanik der Organismen. Bd. I.

c) Methoden zur Hervorbringung halber Froschembryonen usw. Anatomischer Anzeiger. Bd. 9, S. 260.

1895. Gesammelte Abhandlungen übér Entwicklungsmechanik der Organismen. Bd. I und II. Leipzig.

1896. a) Über die Selbstordnung (Cytotaxis) sich berührender Furchungszellen des Froscheies durch Zellenzusammenfiugung, Zellentrennung und Zellengleiten. Dies Archiv. Bd. III.

b) Über die Bedentung *geringer * Verschiedenheiten der relativen Größe der Furchungszellen für den Charakter des Furchungsschemas nebst Erörterung über die nächsten Ursachen der Anordnung und Gestalt der ersten Furchungszellen. Dies Archiv Bd. IV.

1902. Verhandlungen der anatomischen Gesellschaft.

1911. Die vier kausalen Hauptperioden der Ontogenese, sowie das doppelte Bestimmtsein der organischen Gestaltungen. Mitteil. d. Naturforschd. Ges. zu Halle a. S. Bd. 1.

1912. Terminologie der Entwicklungsmechanik der Tiere und Pflanzen. Leipzig. $465 \mathrm{~S}$.

1913. Über die bei der Vererbung von Variationen anzunehmenden Vorgänge nebst einer Einschaltung über die Hauptarten des Entwicklungsgeschehens. Vorträge u. Anfsätze üb. Entw.-Mech. Nr. 19. Leipzig. $68 \mathrm{~S}$.

1915. Das Wesen des Lebens. Artikel in: Die Kultar der Gegenwart, Band Allgemeine Biologie. S. 173-187. 